\title{
Investigation of the Hall-Petch Relationship Using Strain Gradient Plasticity Model for Finite Deformation Framework
}

\author{
Yooseob Song \\ Department of Civil Engineering, The University of Texas Rio Grande Valley, Edinburg, TX 78539, USA \\ E-mail: yooseob.song@utrgv.edu
}

Received: 6 April 2020; Accepted: 27 April 2020; Available online:25 June 2020

\begin{abstract}
The Hall-Petch relationship in metals is investigated using the strain gradient plasticity theory within the finite deformation framework. For this purpose, the thermodynamically consistent constitutive formulation for the coupled thermomechanical gradient-enhanced plasticity model is developed. The corresponding finite element method is performed to investigate the characteristics of the Hall-Petch relationship in metals. The proposed model is established based on an extra Helmholtz-type partial differential equation, and the nonlocal quantity is calculated in a coupled method based on the equilibrium conditions. An excellent agreement between the simulation results and the test data is resulted in the Hall-Petch graph. Furthermore, it is observed that the Hall-Petch constants do not remain unchanged but vary with the strain level.
\end{abstract}

Keywords: Hall-Petch; Strain gradient plasticity; Finite deformation; Finite element simulations.

\section{Introduction}

Most of metals and metal alloys have polycrystalline nature. In general, a fine-grained material is stronger and harder than a coarse-grained one. This can be described in the relation between grain size and yield stress through the Hall-Petch equation as follow [1, 2]:

$$
\sigma_{y}=\sigma_{0}+\frac{k}{\sqrt{D}}
$$

where $\sigma_{y}$ denotes the yield stress, $\sigma_{0}$ denotes the material constant related to the resistance of lattice to dislocation motion, $k$ denotes the Hall-Petch strengthening coefficient, and $D$ denotes the average grain size. A linear relationship between $\sigma_{y}$ and $D^{-1 / 2}$ with a slope of $k$ is shown in the Hall-Petch plot.

After the pioneering works of Hall [1] for mild steels and Petch [2] for brittle materials, numerous works have been conducted to investigate the Hall-Petch relation through various methods including experiments [3]; review/overview [4, 5]; theoretical investigations [6]; numerical simulations [7]. In [3], microhardness of nanocrystalline palladium and copper were experimentally investigated according to grain size variation and significant increases in strength were observed in both materials compared to conventional grain size materials.

However, strain gradient continuum plasticity is rarely used in this area. Song and his co-workers [8-16] have developed the coupled thermo-mechanical and thermodynamically consistent strain gradient plasticity models to study the characteristics of nano/micro-scale metallic materials. In this work, strain gradient-enhanced flow rules are proposed to investigate the grain-size dependent flow stress of polycrystalline materials. The implicit gradientenhanced theory (IGT) is introduced in this work. In the IGT, the Helmholtz-type partial differential equation (PDE) is obtained, and the nonlocal quantities are calculated in a coupled method based on the equilibrium conditions.

The main aim of this work is to show that the proposed strain gradient-enhanced flow rule well captures the Hall-Petch relation. The two-dimensional finite element solution for finite deformation with considering the temperature effect is used to investigate the Hall-Petch relationship.

\section{Theoretical development}

\subsection{Kinematics}

The homogeneous body $\mathfrak{B}$ in the reference (undeformed), intermediate and current (deformed) states is presented. The material point is simply denoted by $\boldsymbol{X}$, and its Lagrangian coordinates are denoted by $X_{i}(i=$ 
$1,2,3)$. The point in the current state is denoted by $\boldsymbol{x}$, and its Eulerian coordinates are denoted by $x_{i}(i=1,2,3)$. In this figure, the one to one mapping function $\boldsymbol{x}=\boldsymbol{M}(\boldsymbol{X}, t)$ is used for defining the velocity $\boldsymbol{v}$ and the deformation gradient $\boldsymbol{F}$ respectively such as $v_{i}=\partial \mathcal{M}_{i}\left(X_{i}, t\right) / \partial t=\dot{\mathcal{M}}_{i}$ and $F_{i j}=\partial x_{i} / \partial X_{j}=\partial \mathcal{M}_{i}\left(X_{i}, t\right) / \partial X_{j}$. In this work, the following multiplicative decomposition is employed

$$
d x_{i}=\underbrace{F_{i k}^{e} F_{k j}^{p}}_{F_{i j}} d X_{j}
$$

where $\boldsymbol{F}$ is the deformation gradient, $\boldsymbol{F}^{e}$ is the elastic distortion and $\boldsymbol{F}^{p}$ is the plastic distortion. $\boldsymbol{F}^{p}$ indicates the local plastic deformation of the material at $\boldsymbol{X}$ due to the plastic mechanism in the microscopic neighborhood of $\boldsymbol{X}$. This local deformation takes the material into (and ultimately pins the material to) a coherent structure that resides in the structural space at $\boldsymbol{X}$. The subsequent rotation and stretching of this coherent structure are represented by the non-symmetric tensor $\boldsymbol{F}^{e}$, thus $\boldsymbol{F}^{e}$ denotes the elastic mechanism.

The implicit function theorem $\boldsymbol{F}$ must be non-singular, that is, the Jacobian determinant $J(\boldsymbol{X}, t) \stackrel{\text { def }}{=}$ $\operatorname{det} \boldsymbol{F}(\boldsymbol{X}, t) \neq 0$. The Jacobian determinant is the property of measuring the ratio between deformed $(\Omega)$ and undeformed $\left(\Omega_{0}\right)$ infinitesimal volumes, that is,

$$
d \Omega=J d \Omega_{0}
$$

The velocity gradient is given by

$$
\begin{aligned}
L_{i j}=\frac{\partial \dot{u}_{i}}{\partial x_{j}}=\dot{F}_{i k} F_{k j}^{-1} & =\overbrace{\dot{F}_{i k}^{e} F_{k j}^{e^{-1}}}^{L_{i j}^{e \text { def }}}+F_{i k}^{e} \overbrace{\dot{F}_{k l}^{p} F_{l m}^{p^{-1}}}^{L_{k m}^{p}} F_{m j}^{e^{-1}} \\
= & \left(D_{i j}^{e}+W_{i j}^{e}\right)+F_{i k}^{e}\left(D_{k m}^{p}+W_{k m}^{p}\right) F_{m j}^{e^{-1}}
\end{aligned}
$$

where $\boldsymbol{L}^{e}$ and $\boldsymbol{L}^{p}$ represent the elastic and plastic distortion rate tensors addressed with the elastic and plastic components of the deformation rate tensor $\boldsymbol{D}$ and the spin rate tensor $\boldsymbol{W}$, that is, $\boldsymbol{L}^{e}=\boldsymbol{D}^{e}+\boldsymbol{W}^{e}$ and $\boldsymbol{L}^{p}=\boldsymbol{D}^{p}+$ $\boldsymbol{W}^{p}$. The elastic and plastic parts of $\boldsymbol{D}$ and $\boldsymbol{W}$ are given by

$$
D_{i j}^{e}=\operatorname{sym} L_{i j}^{e}, \quad D_{i j}^{p}=\operatorname{sym} L_{i j}^{p}, \quad W_{i j}^{e}=\operatorname{asym} L_{i j}^{e}, \quad W_{i j}^{p}=\operatorname{asym} L_{i j}^{p}
$$

Note that the plastic flow does not induce changes in volume, consistently $\operatorname{det} \boldsymbol{F}^{p}=1$, and $\boldsymbol{L}^{p}$ and $\boldsymbol{D}^{p}$ are deviatoric.

The right polar decomposition of a second-order, non-singular tensor $\boldsymbol{F}^{e}$ can be obtained by the orthogonal rotation tensor $\boldsymbol{R}^{e}$ and the symmetric, positive definite right stretch tensor $\boldsymbol{U}^{e}$ as

$$
F_{i j}^{e}=R_{i k}^{e} U_{k j}^{e}
$$

where $\boldsymbol{U}^{e}=\sqrt{\boldsymbol{F}^{e^{T}} \boldsymbol{F}^{e}}$.

The elastic Cauchy-Green deformation tensor $\boldsymbol{C}^{e}$ can be given by

$$
C_{i j}^{e}=F_{k i}^{e} F_{k j}^{e}=U_{l i}^{e} U_{l j}^{e}
$$

Then, the material time derivative of $\boldsymbol{C}^{e}$ can be obtained by differentiating Eq. (7) as follows:

$$
\dot{C}_{i j}^{e}=\dot{F}_{k i}^{e} F_{k j}^{e}+F_{k i}^{e} \dot{F}_{k j}^{e}=2 \operatorname{sym}\left(F_{k i}^{e} \dot{F}_{k j}^{e}\right)=2 \dot{E}_{i j}^{e}
$$

where $\dot{\boldsymbol{E}}^{e}$ represents the rate of elastic strain $\boldsymbol{E}^{e}$.

\subsection{Nonlocal effective plastic strain and Helmholtz-type partial differential equation}

The IGT is used in this work because of its algorithmic efficiency compared to other gradient-enhanced approaches. The nonlocal effective plastic strain $\bar{\varepsilon}^{p}$, which indicates a weighted average of the local effective plastic strain $\varepsilon^{p}$, is introduced and obtained by the following PDE with the material length scale parameter $l_{p}$ :

$$
\bar{\varepsilon}^{p}-l_{p}^{2} \nabla^{2} \bar{\varepsilon}^{p}=\varepsilon^{p}
$$




\subsection{Coupled thermo-mechanical framework}

\subsubsection{Principle of virtual power}

The principle of virtual power has been widely employed to formulate a thermodynamically consistent strain gradient plasticity theory and to derive the microforce balances, e.g. [17-19]. Song and his co-workers [8-16] have also successfully developed the gradient-enhanced models by incorporating the thermal effect based on the principle of virtual power to investigate the behavior of small-scale metallic materials. The power expended by microstress quantities along with time-based changes in plastic strain and its gradient will be accounted for, which naturally leads to a microforce balances that formulate the flow rule by means of thermodynamically consistent constitutive equations. In this section, the fully coupled thermo-mechanical theory is formulated by considering a nonlocal variable $\overline{\varepsilon_{p}}$ in conjunction with the new forms of the dissipation potential and free energy function.

The elastic deformation power in the deformed state $\mathcal{P}_{i n t}^{e}$ can be given with the Cauchy stress tensor $\boldsymbol{\sigma}$ and the deformation rate tensor $\boldsymbol{D}$ by

$$
\mathcal{P}_{\text {int }}^{e}=\int_{\bar{\Omega}} \sigma_{i j} D_{i j} d \Omega
$$

Since $\boldsymbol{\sigma}$ is symmetric, $\boldsymbol{\sigma}: \boldsymbol{D}=\boldsymbol{\sigma}: \boldsymbol{L}$. Then, by using Eq. (4), $\boldsymbol{\sigma}: \boldsymbol{L}=\operatorname{tr} \boldsymbol{\sigma} \dot{\boldsymbol{F}} \boldsymbol{F}^{-1}=\left(\boldsymbol{\sigma} \boldsymbol{F}^{-T}\right): \dot{\boldsymbol{F}}$. Now, $\mathcal{P}_{\text {int }}^{e}$ can be expressed alternatively by using Eq. (3) as follows:

$$
\mathcal{P}_{i n t}^{e}=\int_{\bar{\Omega}_{0}} \overbrace{J \sigma_{i k} F_{j k}^{-1}}^{P_{i j}^{\text {def }}} \dot{F}_{i j} d \Omega_{0}=\int_{\bar{\Omega}_{0}} P_{i j} \dot{F}_{i j} d \Omega_{0}
$$

where the non-symmetric first Piola-Kirchhoff stress tensor $\boldsymbol{P}$ can be obtained by performing a Piola transformation on the symmetric Cauchy stress tensor $\boldsymbol{\sigma}$. Here, the first Piola-Kirchhoff stress $\boldsymbol{P}$ is defined using another stress quantity $\boldsymbol{\sigma}^{e}$ (hereafter, written as the elastic stress) as follows:

$$
P_{i j} \stackrel{\text { def }}{=} \sigma_{i l}^{e} F_{j l}^{p-1}
$$
$\sigma^{e}$

Moreover, the substitution of Eq. (11) into Eq. (12) gives the following equation for the elastic stress quantity

$$
\sigma_{i j}^{e}=J \sigma_{i k} F_{j k}^{e^{-1}}
$$

Meanwhile, by using Eqs. (11) and (12), the elastic deformation power is expressed as $\int_{\bar{\Omega}_{0}} \sigma_{i j}^{e} \dot{F}_{i j}^{e} d \Omega_{0}$. Finally, the internal power $\mathcal{P}_{\text {int }}$ can be written with energy contributions as follows:

$$
\mathcal{P}_{\text {int }}=\int_{\bar{\Omega}_{0}}(\underbrace{\sigma_{i j}^{e} \dot{F}_{i j}^{e}}_{\text {Macro- }}+\underbrace{x \dot{\varepsilon}^{p}+q \dot{\bar{\varepsilon}}^{p}+\mathcal{Q}_{i} \dot{\bar{\varepsilon}}_{, i}^{p}}_{\text {Micro- }}+\underbrace{\mathcal{A} \dot{\mathcal{T}}+\mathcal{B}_{i} \dot{\mathcal{T}}_{, i}}_{\text {Thermal }}) d \Omega_{0}
$$

where $\bar{\Omega}_{0}$ is an arbitrary subregion of the reference body $\Omega_{0}, \dot{\boldsymbol{F}}^{e}$ is the elastic distortion rate, $x, \boldsymbol{q}$ and $\boldsymbol{Q}$ are the microscopic stresses conjugate to the local effective plastic strain rate $\dot{\varepsilon}^{p}$, nonlocal effective plastic strain rate $\dot{\bar{\varepsilon}}^{p}$ and the gradient of $\dot{\bar{\varepsilon}}^{p}$ respectively. $\mathcal{A}$ and $\mathcal{B}$ in the last two terms are the generalized stresses and are related to the temperature $\mathcal{T}$.

The external power $\mathcal{P}_{\text {ext }}$ is given with the generalized external body forces $\boldsymbol{b}$ acting within $\bar{\Omega}_{0}$ and the macrotraction $\boldsymbol{t}$ and micro-traction $m$ on the external surface $\partial \bar{\Omega}_{0}$ as follows:

$$
\mathcal{P}_{\text {ext }}=\int_{\bar{\Omega}_{0}}(\underbrace{b_{i} \dot{\mathcal{M}}_{i}}_{\text {Macro }-}) d \Omega_{0}+\int_{\partial \bar{\Omega}_{0}}(\underbrace{t_{i} \dot{\mathcal{M}}_{i}}_{\text {Macro- }}+\underbrace{m \dot{\bar{\varepsilon}}^{p}}_{\text {Micro- }}+\underbrace{a \dot{\mathcal{T}}}_{\text {Thermal }}) d S_{0}
$$

For the thermal effect, the external virtual power is assumed to have the term of $a$ conjugate to $\dot{\mathcal{T}}$.

Meanwhile, by the assumption of the irrotational plastic flow $\left(\boldsymbol{W}^{p}=\mathbf{0}\right)$ and the definition of the mapping function $\boldsymbol{M}(\boldsymbol{X}, t)$, Eq. (4) can be expressed as 


$$
\dot{\mathcal{M}}_{i, k} F_{k j}^{-1}=\dot{F}_{i k}^{e} F_{k j}^{e^{-1}}+F_{i k}^{e} D_{k m}^{p} F_{m j}^{e^{-1}}
$$

Using the assumption that $\varepsilon^{p}$ evolves along the plastic stretch, i.e. $\dot{\varepsilon}^{p}=\left|D_{i j}^{p}\right|$ with an initial condition $\varepsilon^{p}(\boldsymbol{X}, 0)=0$ and the definition of the plastic flow direction by $N_{i j}^{p}=D_{i j}^{p} /\left|D_{i j}^{p}\right|=\bar{M}_{i j}^{e} /\left|\bar{M}_{i j}^{e}\right|$ with whenever $\left|D_{i j}^{p}\right| \neq 0$, Eq. (16) can be expressed as

$$
\dot{\mathcal{M}}_{i, k} F_{k j}^{-1}=\dot{F}_{i k}^{e} F_{k j}^{e^{-1}}+\dot{\varepsilon}^{p} F_{i k}^{e} N_{k m}^{p} F_{m j}^{e^{-1}}
$$

In the definition of the plastic flow direction $\boldsymbol{N}^{p}$, the term $\boldsymbol{M}^{e}$ represents the elastic Mandel stress and is expressed later by Eqs. (34) and (37). $\overline{\boldsymbol{M}}^{e}$ is the deviatoric part of the Mandel stress, i.e. $\bar{M}_{i j}^{e}=M_{i j}^{e}-M_{k k}^{e} \delta_{i j} / 3$.

By considering $\mathcal{M}, \dot{\boldsymbol{F}}^{e}, \dot{\varepsilon}^{p}, \dot{\bar{\varepsilon}}^{p}$ and $\dot{T}$ as the virtual velocity fields with the variations of $\delta \boldsymbol{M}, \delta \boldsymbol{F}^{e}, \delta \varepsilon^{p}, \delta \bar{\varepsilon}^{p}$ and $\delta T$ respectively, the internal and external parts of the virtual power are written from Eqs. (14) and (15) as follows:

$$
\begin{gathered}
\mathcal{P}_{\text {int }}^{\text {vir }}=\int_{\bar{\Omega}_{0}}\left(\sigma_{i j}^{e} \delta F_{i j}^{e}+x \delta \varepsilon^{p}+q \delta \bar{\varepsilon}^{p}+\mathcal{Q}_{i} \delta \bar{\varepsilon}_{, i}^{p}+\mathcal{A} \delta T+\mathcal{B}_{i} \delta T_{, i}\right) d \Omega_{0} \\
\mathcal{P}_{\text {ext }}^{\text {vir }}=\int_{\bar{\Omega}_{0}}\left(b_{i} \delta \mathcal{M}_{i}\right) d \Omega_{0}+\int_{\partial \bar{\Omega}_{0}}\left(t_{i} \delta \mathcal{M}_{i}+m \delta \bar{\varepsilon}^{p}+a \delta T\right) d S_{0}
\end{gathered}
$$

and Eq. (17) becomes

$$
\delta \mathcal{M}_{i, k} F_{k j}^{-1}=\delta F_{i k}^{e} F_{k j}^{e^{-1}}+\delta \varepsilon^{p} F_{i k}^{e} N_{k m}^{p} F_{m j}^{e^{-1}}
$$

The following relation is established in an arbitrary subregion $\bar{\Omega}_{0}$ by the fundamental statement of the principle of virtual power:

$$
\mathcal{P}_{\text {int }}^{\text {vir }}=\mathcal{P}_{\text {ext }}^{\text {vir }}
$$

\subsubsection{Objectivity of the internal power}

Substituting Eq. (2) into the equations, $F_{i j}^{\prime}=R_{i k} F_{k j}$ where $\boldsymbol{R}$ is the rotational transformation matrix, gives

$$
F_{i k}^{e{ }^{\prime}} F_{k j}^{p^{\prime}}=R_{i k} F_{k l}^{e} F_{l j}^{p}
$$

The reference and intermediate states are independent of the changes in frame,

$$
F_{i j}^{e^{\prime}}=R_{i k} F_{k j}^{e}
$$

and $\boldsymbol{F}^{p}$ is objective. Differentiating Eq. (23) gives

$$
\dot{F}_{i j}^{e^{\prime}}=\dot{R}_{i k} F_{k j}^{e}+R_{i k} \dot{F}_{k j}^{e}
$$

Similarly, $\boldsymbol{L}^{p}, \boldsymbol{D}^{p}, \boldsymbol{U}^{e}$ (hence $\boldsymbol{E}^{e}$ ) and $\boldsymbol{C}^{e}$ are objective under a change in frame. This consequence of frame invariance is reserved for later use. Now, assume that $\mathcal{P}_{\text {int }}^{\text {vir }}=\mathcal{P}_{\text {int }}^{\text {vir }}$. Under a change in frame, $x, q, \mathcal{A}, \delta \varepsilon^{p}, \delta \bar{\varepsilon}^{p}$ and $\delta \mathcal{T}$ in Eq. (18) are invariant because all of these terms are scalar fields. $\delta \bar{\varepsilon}_{, i}^{p}$ and $\delta \mathcal{T}_{, i}$ also remain unchanged since they are a gradient of $\delta \varepsilon^{p}$ and $\delta \mathcal{T}$ in the reference state. On the other hand, $\boldsymbol{\sigma}^{e}, \boldsymbol{Q}$ and $\boldsymbol{B}$ are converted to $\boldsymbol{\sigma}^{e^{\prime}}$, $\boldsymbol{R}^{\prime}$ and $\boldsymbol{B}^{\prime}$ respectively, and using Eq. (24), $\delta \boldsymbol{F}^{e}$ is converted to

$$
\delta F_{i j}^{e^{\prime}}=\dot{R}_{i k} F_{k j}^{e}+R_{i k} \delta F_{k j}^{e}=R_{i k}\left(R_{k i} \dot{R}_{i k} F_{k j}^{e}+\delta F_{k j}^{e}\right)
$$

From the assumption $\mathcal{P}_{\text {int }}^{\text {vir }}=\mathcal{P}_{\text {int }}^{\text {vir }}$, it can be easily shown that the stress quantities $\mathcal{Q}_{i}$ and $\mathcal{B}_{i}$ are invariant. Under the assumption that $\boldsymbol{R}$ is an arbitrary time-independent transformation $(\dot{\boldsymbol{R}}=\mathbf{0})$, the transformation of $\boldsymbol{\sigma}^{e}$ can be obtained by 


$$
\sigma_{i j}^{e \prime}=R_{i k} \sigma_{k j}^{e}
$$

On the other hand, if one assumes $\boldsymbol{R}=\boldsymbol{I}$, after some algebraic manipulation, the following equation is obtained:

$$
\sigma_{i j}^{e} F_{j k}^{e} \dot{R}_{i k}=0
$$

which indicates that $\boldsymbol{\sigma}^{e} \boldsymbol{F}^{e^{T}}=\boldsymbol{F}^{e} \boldsymbol{\sigma}^{e T}$.

\subsubsection{Balance equations}

By assuming $\delta \bar{\varepsilon}^{p}=0$ and $\delta \varepsilon^{p}=0$, and using the description of the tensor $\boldsymbol{P}$ given in Eq. (11), the equations for the local macroscopic surface traction conditions, the balance of the macroscopic linear momentum and the balance of the generalized stresses $\mathcal{A}$ and $\mathcal{B}$ (under equilibrium) can be represented respectively as follows:

$$
\begin{gathered}
t_{i}=P_{i j} n_{j} \\
P_{i j, j}+b_{i}=0_{i} \\
a=\mathcal{B}_{i} n_{i} \\
\mathcal{B}_{i, i}-\mathcal{A}=0
\end{gathered}
$$

where $\boldsymbol{n}$ is the outward unit normal vector to $\partial \bar{\Omega}_{0}$.

Next, by assuming $\delta \mathcal{M}=\mathbf{0}, \delta \varepsilon^{p}=0$ and $\delta \mathcal{T}=0$, and after some algebraic manipulations, the equations for the first microscopic force balance and the nonlocal microscopic traction conditions are given by

$$
\begin{gathered}
\mathcal{Q}_{i, i}-q=0 \\
m=\mathcal{Q}_{i} n_{i}
\end{gathered}
$$

Lastly, by assuming $\delta \mathcal{M}=\mathbf{0}, \delta \bar{\varepsilon}^{p}=0$ and $\delta \mathcal{T}=0$, and defining

$$
\begin{gathered}
M_{i j}^{e} \stackrel{\text { def }}{=} F_{i k}^{e} \sigma_{k j}^{e} \\
\bar{\sigma} \stackrel{\text { def }}{=} \bar{M}_{i j}^{e} N_{i j}^{p}=\left|\bar{M}_{i j}^{e}\right|
\end{gathered}
$$

The second microscopic force balance can be obtained by

$$
x=\bar{\sigma}
$$

where $\boldsymbol{M}^{e}$ represents the elastic Mandel stress, which is given by

$$
M_{i j}^{e}=J F_{k i}^{e} \sigma_{k l} F_{j l}^{e^{-1}}
$$

$\bar{\sigma}$ denotes an equivalent tensile stress. For more details about this section.

\subsubsection{Laws of thermodynamics}

The first law of thermodynamics is employed in this work as follows:

$$
\rho \dot{e}=\sigma_{i j}^{e} \dot{F}_{i j}^{e}+x \dot{\varepsilon}^{p}+q \dot{\bar{\varepsilon}}^{p}+\mathcal{Q}_{i} \dot{\bar{\varepsilon}}_{, i}^{p}+\mathcal{A} \dot{\mathcal{T}}+\mathcal{B}_{i} \dot{\mathcal{T}}_{, i}-q_{i, i}+\rho \mathcal{H}_{E X T}
$$

where the terms $\boldsymbol{q}, \rho, e$ and $\mathcal{H}_{E X T}$ denote the thermal flux vector, spatial mass density, specific internal energy, and specific heat from the external source respectively. The entropy production inequality is formulated from the second law as follows:

$$
-\rho \dot{e}+\rho \dot{s} \mathcal{T}+\sigma_{i j}^{e} \dot{F}_{i j}^{e}+x \dot{\varepsilon}^{p}+q \dot{\bar{\varepsilon}}^{p}+\mathcal{Q}_{i} \dot{\bar{\varepsilon}}_{, i}^{p}+\mathcal{A} \dot{\mathcal{T}}+\mathcal{B}_{i} \dot{\mathcal{T}}_{, i}-q_{i} \frac{\mathcal{T}_{, i}}{\mathcal{T}} \geq 0
$$

where $s$ denotes the specific entropy. 


\subsection{Thermodynamic forces: energetic and dissipative}

The following Clausius-Duhem inequality is formulated by equating the time derivative of the Helmholtz free energy (per unit volume), $\Psi=e-\mathcal{T}$ s, into Eq. (39).

$$
\sigma_{i j}^{e} \dot{F}_{i j}^{e}+x \dot{\varepsilon}^{p}+q \dot{\bar{\varepsilon}}^{p}+Q_{i} \dot{\bar{\varepsilon}}_{, i}^{p}+\mathcal{A} \dot{\mathcal{T}}+\mathcal{B}_{i} \dot{\mathcal{T}}_{, i}-\rho \dot{\Psi}-\rho s \dot{\mathcal{T}}-q_{i} \frac{\mathcal{T}_{i}}{\mathcal{T}} \geq 0
$$

From Eqs. (24) and (26), both $\boldsymbol{\sigma}^{e}$ and $\dot{\boldsymbol{F}}^{e}$ are not objective under a change in frame. Thus, the elastic stress power is most conveniently represented through the objective elastic stress rate $\dot{\boldsymbol{E}}^{e}$. To accompany this, the second Piola-Kirchhoff elastic stress $\boldsymbol{P}^{e}$ is defined such as

$$
P_{i j}^{e} \stackrel{\text { def }}{=} J F_{i k}^{e^{-1}} \sigma_{k l} F_{j l}^{e^{-1}}
$$

Accordingly, by Eq. (13), $\boldsymbol{P}^{e}$ can be expressed with $\boldsymbol{\sigma}^{e}$ as

$$
P_{i j}^{e}=F_{i k}^{e^{-1}} \sigma_{k j}^{e}
$$

Note that by using the definitions of $\boldsymbol{M}^{e}$ and $\boldsymbol{P}^{e}$ given respectively in Eqs. (37) and (41), the relationship between $\boldsymbol{M}^{e}$ and $\boldsymbol{P}^{e}$ can be obtained by

$$
M_{i j}^{e}=C_{i k}^{e} P_{k j}^{e}
$$

Eqs. (23), (26), (27) and (42) yield that $\boldsymbol{P}^{e}$ is symmetric and objective under a change in frame. Finally, the elastic stress power $\boldsymbol{\sigma}^{e}: \dot{\boldsymbol{F}}^{e}$ can be converted to the new form with the objective tensors $\boldsymbol{P}^{e}$ and $\dot{\boldsymbol{E}}^{e}$ as follows:

$$
\sigma_{i j}^{e} \dot{F}_{i j}^{e}=F_{i k}^{e} P_{k j}^{e} \dot{F}_{i j}^{e}=P_{k j}^{e}\left(F_{k i}^{e} \dot{F}_{i j}^{e}\right)=P_{k j}^{e} \dot{E}_{k j}^{e}
$$

Substituting Eq. (44) into Eq. (40) gives that

$$
P_{i j}^{e} \dot{E}_{i j}^{e}+x \dot{\varepsilon}^{p}+q \dot{\bar{\varepsilon}}^{p}+Q_{i} \dot{\bar{\varepsilon}}_{, i}^{p}+\mathcal{A} \dot{\mathcal{T}}+\mathcal{B}_{i} \dot{\mathcal{T}}_{, i}-\rho \dot{\Psi}-\rho s \dot{\mathcal{T}}-q_{i} \frac{\mathcal{T}_{i}}{\mathcal{T}} \geq 0
$$

Meanwhile, to model the small-scale phenomena including the effect of size on the material mechanical properties and the width of the localization zones in the softening media in the localized region, an attempt is made in this work to account for the effect of nonuniform distribution of microdefects on the homogenized response of the material. The functional form of the Helmholtz free energy $\Psi$ in terms of its state variables is defined as

$$
\Psi=\Psi\left(E_{i j}^{e}, \varepsilon^{p}, \bar{\varepsilon}^{p}, \bar{\varepsilon}_{, i}^{p}, \mathcal{T}, \mathcal{T}_{i}\right)
$$

where $\Psi$ is assumed to be a smooth function.

Note that the plastic dissipation work must be non-negative in the development process of the constitutive equations. By using Eqs. (45) and (46), one can obtain the following inequality:

$$
\begin{gathered}
\left(P_{i j}^{e}-\rho \frac{\partial \Psi}{\partial E_{i j}^{e}}\right) \dot{E}_{i j}^{e}+\left(x-\rho \frac{\partial \Psi}{\partial \varepsilon^{p}}\right) \dot{\varepsilon}^{p}+\left(q-\rho \frac{\partial \Psi}{\partial \bar{\varepsilon}^{p}}\right) \dot{\bar{\varepsilon}}^{p}+\left(\mathcal{Q}_{i}-\rho \frac{\partial \Psi}{\partial \bar{\varepsilon}_{, i}^{p}}\right) \dot{\bar{\varepsilon}}_{, i}^{p} \\
+\left(\mathcal{A}-\rho s-\rho \frac{\partial \Psi}{\partial \mathcal{T}}\right) \dot{\mathcal{T}}+\left(\mathcal{B}_{i}-\rho \frac{\partial \Psi}{\partial \mathcal{T}_{i}}\right) \dot{\mathcal{T}}_{, i}-\frac{q_{i}}{\mathcal{T}} \mathcal{T}_{, i} \geq 0
\end{gathered}
$$

One now assumes that the thermodynamic conjugate microstress quantities $x, q, Q_{i}$ and $\mathcal{A}$ are decomposed into the energetic and the dissipative components $[8,10,14,19,20]$.

$$
x=x^{e n}+x^{d i s} ; q=q^{e n}+q^{d i s} ; Q_{i}=Q_{i}^{e n}+Q_{i}^{d i s} ; \mathcal{A}=\mathcal{A}^{e n}+\mathcal{A}^{d i s}
$$

By using Eqs. (47) and (48), the energetic microstresses are defined as follows: 


$$
P_{i j}^{e}=\rho \frac{\partial \Psi}{\partial E_{i j}^{e}} ; x^{e n}=\rho \frac{\partial \Psi}{\partial \varepsilon^{p}}: q^{e n}=\rho \frac{\partial \Psi}{\partial \bar{\varepsilon}^{p}} ; \mathcal{Q}_{i}^{e n}=\rho \frac{\partial \Psi}{\partial \bar{\varepsilon}_{, i}^{p}} ; \mathcal{A}^{e n}=\rho\left(s+\frac{\partial \Psi}{\partial \mathcal{T}}\right) ; \mathcal{B}_{i}=\rho \frac{\partial \Psi}{\partial \mathcal{T}_{, i}}
$$

Therefore the dissipation potential $\mathcal{D}$ can be obtained as

$$
\mathcal{D}=x^{d i s} \dot{\varepsilon}^{p}+q^{d i s} \dot{\bar{\varepsilon}}^{p}+\mathcal{Q}_{i}^{d i s} \dot{\bar{\varepsilon}}_{, i}^{p}+\mathcal{A}^{d i s} \dot{\mathcal{T}}-\frac{q_{i}}{\mathcal{T}} \mathcal{T}_{, i} \geq 0
$$

The dissipative microstresses can then be obtained from $\mathcal{D}=\mathcal{D}\left(\dot{\varepsilon}^{p}, \dot{\bar{\varepsilon}}^{p}, \dot{\bar{\varepsilon}}_{, i}^{p}, \dot{\mathcal{T}}, \mathcal{T}_{, i}\right)$ as follows:

$$
x^{d i s}=\frac{\partial \mathcal{D}}{\partial \dot{\varepsilon}^{p}}: q^{d i s}=\frac{\partial \mathcal{D}}{\partial \dot{\bar{\varepsilon}}^{p}} ; \mathcal{Q}_{i}^{\text {dis }}=\frac{\partial \mathcal{D}}{\partial \dot{\bar{\varepsilon}}_{, i}^{p}} ; \mathcal{A}^{d i s}=\frac{\partial \mathcal{D}}{\partial \dot{\mathcal{T}}} ;-\frac{q_{i}}{\mathcal{T}}=\frac{\partial \mathcal{D}}{\partial \mathcal{T}_{, i}}
$$

\subsection{Helmholtz free energy and energetic thermodynamic forces}

The free energy per unit volume in this work consists of three parts, elastic $\Psi^{e}$, defect $\Psi^{d}$ and thermal $\Psi^{\mathcal{T}}$, as follows:

$$
\Psi\left(E_{i j}^{e}, \varepsilon^{p}, \bar{\varepsilon}^{p}, \bar{\varepsilon}_{, i}^{p}, \mathcal{T}, \mathcal{T}_{, i}\right)=\Psi^{e}\left(E_{i j}^{e}, \mathcal{T}\right)+\Psi^{d}\left(\varepsilon^{p}, \bar{\varepsilon}^{p}, \bar{\varepsilon}_{, i}^{p}, \mathcal{T}\right)+\Psi^{\mathcal{T}}\left(\mathcal{T}, \mathcal{T}_{, i}\right)
$$

\subsubsection{Elastic free energy}

The constitutive equations take a special form when the continuum is isotropic in the undeformed state. In such a case, the elastic free energy density function $\Psi^{e}$ depends on $\boldsymbol{E}^{e}$ through a set of the principal invariants of $\boldsymbol{C}^{e}$ such as

$$
\Psi^{e}=\widehat{\Psi}^{e}\left(I_{C}, I I_{C}, I I I_{C}, \mathcal{T}\right)=\widetilde{\Psi}^{e}\left(\lambda_{1}^{e}, \lambda_{2}^{e}, \lambda_{3}^{e}, \mathcal{T}\right)=\widetilde{\Psi}^{e}\left(E_{1}^{e}, E_{2}^{e}, E_{3}^{e}, \mathcal{T}\right)
$$

where $\lambda_{\alpha}^{e}$ and $E_{\alpha}^{e}(\alpha=1,2,3)$ indicate the eigenvalues of $\boldsymbol{U}^{e}$ and $\boldsymbol{E}^{e}$ respectively, and the invariants $I_{C}, I I_{C}$ and $I I I_{C}$ are given by

$$
\begin{gathered}
I_{C}=\operatorname{tr} \boldsymbol{C}^{e}=C_{k k}^{e} \\
I I_{C}=\frac{1}{2}\left[\left(\operatorname{tr} \boldsymbol{C}^{e}\right)^{2}-\operatorname{tr} \boldsymbol{C}^{e^{2}}\right]=\frac{1}{2}\left[C_{i i}^{e} C_{j j}^{e}-C_{i j}^{e} C_{j i}^{e}\right] \\
I I I_{C}=\operatorname{det} \boldsymbol{C}^{e}=\frac{1}{6} \epsilon_{i j k} \epsilon_{l m n} C_{i l}^{e} C_{j m}^{e} C_{k n}^{e}
\end{gathered}
$$

The spectral decompositions of $\boldsymbol{C}^{e}$ and $\boldsymbol{U}^{e}$ are given by

$$
\begin{aligned}
& \boldsymbol{C}^{e}=\sum_{\alpha=1}^{3} \lambda_{\alpha}^{e^{2}} \boldsymbol{N}_{\alpha}^{e} \otimes \boldsymbol{N}_{\alpha}^{e} \\
& \boldsymbol{U}^{e}=\sum_{\alpha=1}^{3} \lambda_{\alpha}^{e} \boldsymbol{N}_{\alpha}^{e} \otimes \boldsymbol{N}_{\alpha}^{e}
\end{aligned}
$$

where $\boldsymbol{N}_{\alpha}^{e}(\alpha=1,2,3)$ are the orthonormal eigenvectors of $\boldsymbol{C}^{e}$.

Applying the chain rule to Eq. (49) $)_{1}$ gives $\boldsymbol{P}^{e}$ as

$$
\boldsymbol{P}^{e}=\rho \frac{\partial \Psi^{e}}{\partial \boldsymbol{E}^{e}}=\rho \sum_{\alpha=1}^{3} \frac{\partial \breve{\Psi}^{e}\left(\lambda_{1}^{e}, \lambda_{2}^{e}, \lambda_{3}^{e}, \mathcal{T}\right)}{\partial \lambda_{\alpha}^{e}} \frac{\partial \lambda_{\alpha}^{e}}{\partial \boldsymbol{C}^{e}} \frac{\partial \boldsymbol{C}^{e}}{\partial \boldsymbol{E}^{e}}
$$

Using the relation $\boldsymbol{C}^{e}=2 \boldsymbol{E}^{e}+\boldsymbol{I}$ and the derivatives of the stretch $\lambda_{\alpha}^{e}$ with respect to $\boldsymbol{C}^{e}$, i.e. $\partial \lambda_{\alpha}^{e} / \partial \boldsymbol{C}^{e}=$ $\left(1 / 2 \lambda_{\alpha}^{e}\right) \boldsymbol{N}_{\alpha}^{e} \otimes \boldsymbol{N}_{\alpha}^{e}$, Eq. (57) becomes 


$$
\boldsymbol{P}^{e}=2 \rho \sum_{\alpha=1}^{3} \frac{\partial \breve{\Psi}^{e}\left(\lambda_{1}^{e}, \lambda_{2}^{e}, \lambda_{3}^{e}, \mathcal{T}\right)}{\partial \lambda_{\alpha}^{e}} \frac{\partial \lambda_{\alpha}^{e}}{\partial \boldsymbol{C}^{e}}=\rho \sum_{\alpha=1}^{3} \frac{1}{\lambda_{\alpha}^{e}} \frac{\partial \breve{\Psi}^{e}\left(\lambda_{1}^{e}, \lambda_{2}^{e}, \lambda_{3}^{e}, \mathcal{T}\right)}{\partial \lambda_{\alpha}^{e}} \boldsymbol{N}_{\alpha}^{e} \otimes \boldsymbol{N}_{\alpha}^{e}
$$

Next, substituting Eqs. (55) and (58) into Eq. (43) gives the following equation of the elastic Mandel stress.

$$
\boldsymbol{M}^{e}=\rho \sum_{\alpha=1}^{3} \lambda_{\alpha}^{e} \frac{\partial \breve{\Psi}^{e}\left(\lambda_{1}^{e}, \lambda_{2}^{e}, \lambda_{3}^{e}, \mathcal{T}\right)}{\partial \lambda_{\alpha}^{e}} \boldsymbol{N}_{\alpha}^{e} \otimes \boldsymbol{N}_{\alpha}^{e}
$$

The definition of elastic strain $\boldsymbol{E}^{e}$ is given in the logarithmic form as follows:

$$
\boldsymbol{E}^{e}=\ln \boldsymbol{U}^{e}=\sum_{\alpha=1}^{3} E_{\alpha}^{e} \boldsymbol{N}_{\alpha}^{e} \otimes \boldsymbol{N}_{\alpha}^{e}
$$

where $E_{\alpha}^{e}$ is related to $\lambda_{\alpha}^{e}$ as follows:

$$
E_{\alpha}^{e}=\ln \lambda_{\alpha}^{e}(\alpha=1,2,3)
$$

From Eq. (59) one obtains,

$$
\boldsymbol{M}^{e}=\rho \sum_{\alpha=1}^{3} \frac{\partial \widetilde{\Psi}^{e}\left(E_{1}^{e}, E_{2}^{e}, E_{3}^{e}, \mathcal{T}\right)}{\partial E_{\alpha}^{e}} \boldsymbol{N}_{\alpha}^{e} \otimes \boldsymbol{N}_{\alpha}^{e}
$$

In metallic materials in general the elastic strains are small. Accordingly, the simple generalization of the classical strain-energy function of infinitesimal isotropic elasticity which uses a logarithmic measure of finite strain $\boldsymbol{E}^{e}$ is used in this work. The following quadratic form of the classical isotropic elastic strain energy function is considered in conjunction with the linear coupled thermo-elastic free energy:

$$
\widetilde{\Psi}^{e}\left(\boldsymbol{E}^{e}, \mathcal{T}\right)=\frac{\mu}{\rho}\left|\overline{\boldsymbol{E}}^{e}\right|^{2}+\frac{\kappa}{2 \rho}\left(\operatorname{tr} \boldsymbol{E}^{e}\right)^{2}-\frac{\kappa \alpha^{t h}}{\rho}\left(\mathcal{T}-\mathcal{T}_{r}\right) \operatorname{tr} \boldsymbol{E}^{e}
$$

where $\overline{\boldsymbol{E}}^{e}$ is the deviatoric elastic strain, i.e. $\bar{E}_{i j}^{e}=E_{i j}^{e}-E_{k k}^{e} \delta_{i j} / 3, \mathcal{T}_{r}$ is the reference temperature, $\alpha^{\text {th }}$ is the thermal expansion coefficient, and $\mu>0$ and $\kappa>0$ are the shear and bulk moduli respectively.

The substitution of Eq. (63) into Eq. (62) gives the elastic Mandel stress as follows:

$$
\boldsymbol{M}^{e}=2 \mu \overline{\boldsymbol{E}}^{e}+\kappa\left(\operatorname{tr} \boldsymbol{E}^{e}\right) \boldsymbol{I}-\kappa \alpha^{\text {th }}\left(\mathcal{T}-\mathcal{T}_{r}\right) \boldsymbol{I}
$$

where $\boldsymbol{I}$ is the identity tensor.

From Eq. (37), the relationships $\boldsymbol{M}^{e}=J \boldsymbol{R}^{e T} \boldsymbol{\sigma} \boldsymbol{R}^{e}$ and $\boldsymbol{M}^{e}=\boldsymbol{M}^{e T}$ are obtained. The Cauchy stress in the deformed state can be obtained from these relationships as follows:

$$
\boldsymbol{\sigma}=J^{-1} \boldsymbol{R}^{e} \boldsymbol{M}^{e} \boldsymbol{R}^{e^{T}}
$$

\subsubsection{Defect free energy}

The coupled thermo-plastic free energy $\Psi^{d}$ is put forward in terms of the generalized strain quantity $\tilde{\varepsilon}^{p}=$ $\left(\varepsilon^{p}-\bar{\varepsilon}^{p}\right)$, hence combines the macro- and micromorphic variables. For incorporating the effect of the material length scale in the isotropic gradient plasticity model, another contribution to the defect free energy is additionally put forward as the quadratic functions of $\tilde{\varepsilon}^{p}$ and $\bar{\varepsilon}_{, i}^{p}$ as follows:

$$
\Psi^{d}\left(\tilde{\varepsilon}^{p}=\left(\varepsilon^{p}-\bar{\varepsilon}^{p}\right), \bar{\varepsilon}_{, i}^{p}, \mathcal{T}\right)=\frac{h_{e n}}{2 \rho} \tilde{\varepsilon}^{p}\left(1-\left(\frac{\mathcal{T}}{\mathcal{T}_{y}}\right)^{n}\right)+\frac{\mu}{2 \rho} l_{p}^{2} \bar{\varepsilon}_{, i}^{p} \bar{\varepsilon}_{, i}^{p}
$$

where $h_{e n}$ is the material parameter related to the nonlocal energetic contribution to the flow resistance, $l_{p}$ is the plastic material length scale parameter and $n$ and $\mathcal{T}_{y}$ are the thermal material parameters. 
By substituting Eq. (66) into Eq. (49), the energetic thermodynamic microstress qunatities $x^{e n}, q^{e n}$ and $\mathcal{Q}_{i}^{e n}$ can be obtained as follows:

$$
\begin{gathered}
x^{e n}=h_{e n} \tilde{\varepsilon}^{p}\left(1-\left(\frac{\mathcal{T}}{\mathcal{T}_{y}}\right)^{n}\right) \\
q^{e n}=-h_{e n} \tilde{\varepsilon}^{p}\left(1-\left(\frac{\mathcal{T}}{\mathcal{T}_{y}}\right)^{n}\right) \\
\mathcal{Q}_{i}^{e n}=\mu l_{p}^{2} \bar{\varepsilon}_{, i}^{p}
\end{gathered}
$$

\subsubsection{Thermal free energy}

The thermal part of the free energy is given by

$$
\Psi^{\mathcal{T}}\left(\mathcal{T}, \mathcal{T}_{, i}\right)=-\frac{1}{2 \rho}\left[\frac{c_{\varepsilon}}{\mathcal{T}_{r}}\left(\mathcal{T}-\mathcal{T}_{r}\right)^{2}+a \mathcal{T}_{, i} \mathcal{T}_{, i}\right]
$$

where $c_{\varepsilon}$ denotes the specific heat capacity at constant stress and $a$ is the material constant.

The thermodynamic forces $\mathcal{A}^{\text {en }}$ and $\mathcal{B}_{i}$ can be obtained using the definitions in Eq. (49) along with the free energy density functions given in Eqs. (63), (66) and (70) as follows:

$$
\begin{gathered}
\mathcal{A}^{e n}=\rho s-\kappa \alpha^{t h}\left(\mathcal{T}-\mathcal{T}_{r}\right) \operatorname{tr} \boldsymbol{E}^{e}-\frac{h_{e n} \tilde{\varepsilon}^{p^{2}}}{2} \frac{n}{\mathcal{T}_{y}}\left(\frac{\mathcal{T}}{\mathcal{T}_{y}}\right)^{n-1}-\frac{c_{\varepsilon}}{\mathcal{T}_{y}}\left(\mathcal{T}-\mathcal{T}_{r}\right) \\
\mathcal{B}_{i}=-a \mathcal{T}_{, i}
\end{gathered}
$$

\subsection{Dissipation potential and dissipative thermodynamic forces}

The following functional form of the dissipation potential density per unit time is put forward with the assumptions $q^{\text {dis }}=0$ and $\boldsymbol{Q}^{\text {dis }}=\mathbf{0}$.

$$
\begin{aligned}
\mathcal{D}\left(\varepsilon^{p}, \dot{\varepsilon}^{p}, \mathcal{T}, \dot{\mathcal{T}}, \mathcal{T}_{, i}\right) & \\
= & {\left[\sigma_{y}(\mathcal{T})+\left(\sigma_{y}^{s}(\mathcal{T})-\sigma_{y}^{0}(\mathcal{T})\right)\left(1-e^{-\varphi_{1} \varepsilon^{p}}\right)+\mathcal{H}(\mathcal{T}) \varepsilon^{p}+h_{\text {dis }} \tilde{\varepsilon}^{p}\right](1} \\
& \left.-\left(\frac{\mathcal{T}}{\mathcal{T}_{y}}\right)^{n}\right) \dot{\varepsilon}^{p}-\frac{m}{2} \dot{\mathcal{T}}^{2}-\frac{1}{2} \frac{k(\mathcal{T})}{\mathcal{T}} \mathcal{T}_{, i} \mathcal{T}_{, i} \geq 0
\end{aligned}
$$

where $h_{d i s}$ is the material constant for the nonlocal dissipative contribution to the flow resistance, $m$ is the material constant, $k(\mathcal{T})$ is the thermal conductivity coefficient, and $\varphi_{1}$ is the material parameter. The temperature dependent material parameters $\sigma_{y}^{0}(\mathcal{T})>0, \sigma_{y}^{s}(\mathcal{T}) \geq \sigma_{y}^{0}$ and $\mathcal{H}(\mathcal{T}) \geq 0$ are defined as

$$
\begin{aligned}
\sigma_{y}^{0}(\mathcal{T}) & =\sigma_{y}^{0}\left[1-\theta_{0}\left(\mathcal{T}-\mathcal{T}_{r}\right)\right] \\
\sigma_{y}^{s}(\mathcal{T}) & =\sigma_{y}^{s}\left[1-\theta_{\mathcal{H}}\left(\mathcal{T}-\mathcal{T}_{r}\right)\right] \\
\mathcal{H}(\mathcal{T}) & =\mathcal{H}\left[1-\theta_{\mathcal{H}}\left(\mathcal{T}-\mathcal{J}_{r}\right)\right]
\end{aligned}
$$

where $\theta_{0}$ is the flow stress softening parameter and $\theta_{\mathcal{H}}$ is the hardening/softening parameter. The initial yield strength $\sigma_{y}^{0}$ determines the threshold of the elastic response.

Using Eq. (73) along with Eqs. (51) and (74)-(76), and the assumption $k(\mathcal{T}) / \mathcal{T}=k_{0}=$ constant, the dissipative thermodynamic microstress quantities are obtained as

$$
\begin{gathered}
x^{d i s}=\left[\sigma_{y}^{c}\left(\varepsilon^{p}, \mathcal{T}\right)+h_{d i s} \tilde{\varepsilon}^{p}\right]\left(1-\left(\frac{\mathcal{T}}{\mathcal{T}_{y}}\right)^{n}\right)>0 \\
\mathcal{A}^{d i s}=-m \dot{\mathcal{T}} \\
q_{i}=k_{0} \mathcal{T J}_{, i}
\end{gathered}
$$


with

$$
\sigma_{y}^{c}\left(\varepsilon^{p}, \mathcal{T}\right)=\sigma_{y}^{0}(\mathcal{T})+\left(\sigma_{y}^{s}(\mathcal{T})-\sigma_{y}^{0}(\mathcal{T})\right)\left(1-e^{-\varphi_{1} \varepsilon^{p}}\right)+\mathcal{H}(\mathcal{T}) \varepsilon^{p}
$$

A mixed-hardening function $\sigma_{y}^{c}\left(\varepsilon^{p}, \mathcal{T}\right)$ indicates the conventional dissipative strength and it is based on the work by Voce [21]. Particular choices for the material parameters used in Eq. (80) allow for modeling strain hardening, strain softening and strain hardening-softening.

\subsection{Thermo-mechanical coupled heat equation}

In the authors' previous works $[8,10,14]$, thermodynamically consistent and coupled higher-order thermomechanical strain gradient plasticity models were proposed by considering the gradient of temperature and the generalized heat equation.

The first law of thermodynamics in Eq. (38) controls the heat flow generated due to plastic dissipation. By considering Eq. (38) along with Eqs. (49)-(51) and (73), the equation for the entropy evolution can be formulated as follows:

$$
\rho \dot{s} \mathcal{T}=\mathcal{D}+\rho \mathcal{H}_{\text {ext }}
$$

Considering Eq. (71) to solve the rate of the entropy $\dot{s}$ and assuming $c_{v}=$ constant $\cong\left(c_{\varepsilon} \mathcal{T} / \mathcal{T}_{r}\right)$ give the following temperature evolution.

$$
c_{v} \dot{\mathcal{T}}=x^{d i s} \dot{\varepsilon}^{p}-\dot{\mathcal{A}} \mathcal{T}-\kappa \alpha^{t h} \operatorname{tr} \boldsymbol{E}^{e} \dot{\mathcal{T}} \mathcal{T}-\frac{m}{2} \dot{\mathcal{T}}^{2}-\frac{1}{2} \frac{k(\mathcal{T})}{\mathcal{T}} \mathcal{T}_{, i} \mathcal{T}_{, i}+\rho \mathcal{H}_{\text {ext }}
$$

with

$$
\dot{\mathcal{A}}=\dot{\mathcal{A}}^{e n}+h_{e n} \tilde{\varepsilon}^{p} \frac{n}{\mathcal{J}_{y}}\left(\frac{\mathcal{T}}{\mathcal{T}_{y}}\right)^{n-1} \dot{\varepsilon}^{p}+\frac{h_{e n} \tilde{\varepsilon}^{p^{2}}}{2(n-1)} \frac{n}{\mathcal{T}_{y}}\left(\frac{\mathcal{T}}{\mathcal{T}_{y}}\right)^{n-2} \dot{\mathcal{T}}
$$

As can be seen in Eq. (82), the temperature evolution involves the rate of change in energy caused by the irreversible mechanical process, thermo-plastic coupling, thermo-elastic coupling, heat conduction, and the external heat source. Consequently, the temperature evolution can be formulated by substituting the dissipative microforces constitutive equations into Eq. (82) and assuming the absent external heat source as follows:

$$
\begin{aligned}
{\left[c_{v}+\frac{m}{2} \dot{\mathcal{T}}+\kappa \alpha^{t h}\right.} & \left.\operatorname{tr} \boldsymbol{E}^{e} \mathcal{T}+\frac{h_{e n} \tilde{\varepsilon}^{p^{2}}}{2(n-1)} \frac{n}{\mathcal{T}_{y}}\left(\frac{\mathcal{T}}{\mathcal{T}_{y}}\right)^{n-2} \mathcal{T}\right] \dot{\mathcal{T}} \\
= & {\left[\sigma_{y}^{0}(\mathcal{T})+\left(\sigma_{y}^{s}(\mathcal{T})-\sigma_{y}^{0}(\mathcal{T})\right)\left(1-e^{-\varphi_{1} \varepsilon^{p}}\right)+\mathcal{H}(\mathcal{T}) \varepsilon^{p}+h_{d i s} \tilde{\varepsilon}^{p}\right](1} \\
& \left.-\left(\frac{\mathcal{T}}{\mathcal{T}_{y}}\right)^{n}\right) \dot{\varepsilon}^{p}-\left[\dot{\mathcal{A}}^{e n}+h_{e n} \tilde{\varepsilon}^{p} \frac{n}{\mathcal{T}_{y}}\left(\frac{\mathcal{T}}{\mathcal{T}_{y}}\right)^{n-1} \dot{\varepsilon}^{p}\right] \mathcal{T}-\frac{1}{2} \frac{k(\mathcal{T})}{\mathcal{T}} \mathcal{T}_{, i} \mathcal{T}_{, i}
\end{aligned}
$$

\subsection{Yield function}

The following yield criterion is adopted in this work to predict size effect behavior in the strain hardening regime as well as to regularize the localization of plastic deformation during strain softening:

$$
\begin{aligned}
f=\bar{\sigma}-(1-\omega) & {\left[\sigma_{y}^{0}(\mathcal{T})+\left(\sigma_{y}^{S}(\mathcal{T})-\sigma_{y}^{0}(\mathcal{T})\right)\left(1-e^{-\varphi_{1} \varepsilon^{p}}\right)+\mathcal{H}(\mathcal{T}) \varepsilon^{p}+h_{d i s} \tilde{\varepsilon}^{p}\right](1} \\
& \left.-\left(\frac{\mathcal{T}}{\mathcal{T}_{y}}\right)^{n}\right)
\end{aligned}
$$

where $\omega$ is the damage variable which is given by $\omega\left(\bar{\varepsilon}^{p}\right)=1-e^{-\varphi_{2} \bar{\varepsilon}^{p}}$ with the material parameter $\varphi_{2}$. The term $(1-\omega)$ reduces the effective yield stress progressively as $\omega$ increases. This reduction in yield stress leads to a softening behavior and ultimately, the deformation is fully localized on the complete failure (complete loss of local material strength) when $\omega=1$. The softening theory without any gradient effect in hardening can be retrieved by setting $h_{\text {dis }}=0$. 


\subsection{Macroscopic boundary conditions}

The boundary conditions are essential to obtain the finite element solution. In this work, the boundary $(\partial \mathfrak{B})$ of the body (B) is assumed to be divided into two subsurfaces such as $\partial \mathfrak{B}=\partial \mathfrak{B}_{\mathcal{M}} \cup \partial \mathfrak{B}_{t}$ and $\partial \mathfrak{B}_{\mathcal{M}} \cap \partial \mathfrak{B}_{t}=\emptyset$. The following condition for the material motion (mapping function) on $\partial \mathfrak{B}_{\mathcal{M}}$ is considered:

$$
\mathcal{M}=\mathcal{M}^{\star}
$$

and the following surface traction condition is specified on $\partial \mathfrak{B}_{t}$ :

$$
\boldsymbol{P n}=\boldsymbol{t}^{\star}
$$

where $\boldsymbol{M}^{\star}(\boldsymbol{X}, t)$ and $\boldsymbol{t}^{\star}(\boldsymbol{X}, t)$ denote the prescribed functions respectively.

\subsection{Microscopic boundary conditions}

The additional boundary conditions for $\bar{\varepsilon}^{p}$ are required only on the exterior surface region of the body to solve the PDE, Eq. (9). This is in contrast with the explicit gradient-enhanced approach which requires such additional boundary conditions on the elastic-plastic boundary. These boundary conditions are typically applied to the prescribed subsurfaces $\left(\partial \mathfrak{B}_{Q}\right.$ and $\left.\partial \mathfrak{B}_{\bar{\varepsilon}} p\right)$ of the boundary $(\partial \mathfrak{B})$ of the body $(\mathfrak{B})$ in terms of either the normal derivative of $\bar{\varepsilon}^{p}$ or $\bar{\varepsilon}^{p}$ itself.

From the presence of the microstresses $\mathcal{Q}$, the following microscopic power expenditure results: $\int_{\partial \mathfrak{B}}\left(Q_{i} n_{i}\right) \dot{\bar{\varepsilon}}^{p} d A$. Accordingly, the boundary conditions on $\partial \mathfrak{B}\left(\partial \mathfrak{B}=\partial \mathfrak{B}_{\mathcal{Q}} \cup \partial \mathfrak{B}_{\bar{\varepsilon}} p\right.$ and $\left.\partial \mathfrak{B}_{\mathcal{Q}} \cap \partial \mathfrak{B}_{\bar{\varepsilon}^{p}}=\emptyset\right)$, which involve the micro-tractions $Q_{i} n_{i}$ and the nonlocal effective plastic strain rate $\dot{\bar{\varepsilon}}^{p}$, are considered. In particular, special attention is restricted to the boundary conditions which lead to a null power expenditure, i.e. $\left(Q_{i} n_{i}\right) \dot{\bar{\varepsilon}}^{p}=0$. The following equation is taken to be the micro-free boundary condition on a prescribed subsurface $\left(\partial \mathfrak{B}_{Q}\right)$ in this work:

$$
\mathcal{Q}_{i} n_{i}=0
$$

or equivalently, in terms of the constitutive relation given in Eq. (69) along with the assumption $\boldsymbol{Q}^{d i s}=\mathbf{0}$,

$$
\bar{\varepsilon}_{, i}^{p} n_{i}=0
$$

The other condition on $\bar{\varepsilon}^{p}$, which is called the micro-hard boundary condition, is given on the subsurface $\left(\partial \mathfrak{B}_{\bar{\varepsilon}} p\right)$ as follows:

$$
\bar{\varepsilon}^{p}=0
$$

\section{Numerical example: Hall-Petch relationship}

Materials can be strengthened by decreasing the average grain size. This method is called grain boundary strengthening or Hall-Petch strengthening. Grain boundary impedes the movements of dislocations and how many dislocations are existent in a grain affects on how smoothly they can travel from grain to grain. Hall-Petch strengthening is based on this observation. In this section, Hall-Petch strengthening is investigated using finite element simulations with different grain sizes based on the proposed model.

\subsection{Problem description}

The schematic illustration of uniaxial strain problem with single-crystal and polycrystalline is shown in Figure 1. The problem geometry, initial condition, loading condition, macroscopic boundary condition and finite element mesh are displayed in this figure. The term $u^{\dagger}(t)$ represents the prescribed displacement. Each grain has an average grain size of $\mathrm{D}$. The whole square is split into several grains by grain boundaries, which are represented by bold lines, see Figure 2. $4096(64 \times 64)$ elements are used. The general material parameters in Table 1 are used while the rest of the material parameters are calibrated using another set of experiments for pure aluminium (99.999 \%) by [22]. Since the experiments were performed for grain sizes from 0.035 to $1.3 \mathrm{~mm}$ by [22], six different grain sizes, $\mathrm{D}=0.03125 \mathrm{~mm}(32 \times 32=1024$ grains $), \mathrm{D}=0.0625 \mathrm{~mm}(16 \times 16=256$ grains $), \mathrm{D}=0.125 \mathrm{~mm}$ ( $8 \times 8=64$ grains), $\mathrm{D}=0.25 \mathrm{~mm}(4 \times 4=16$ grains $), \mathrm{D}=0.5 \mathrm{~mm}(2 \times 2=4$ grains $)$ and $\mathrm{D}=1.0 \mathrm{~mm}$ (Single grain) are considered in the current simulations. In this work, room temperature is assumed as is in [22]. 

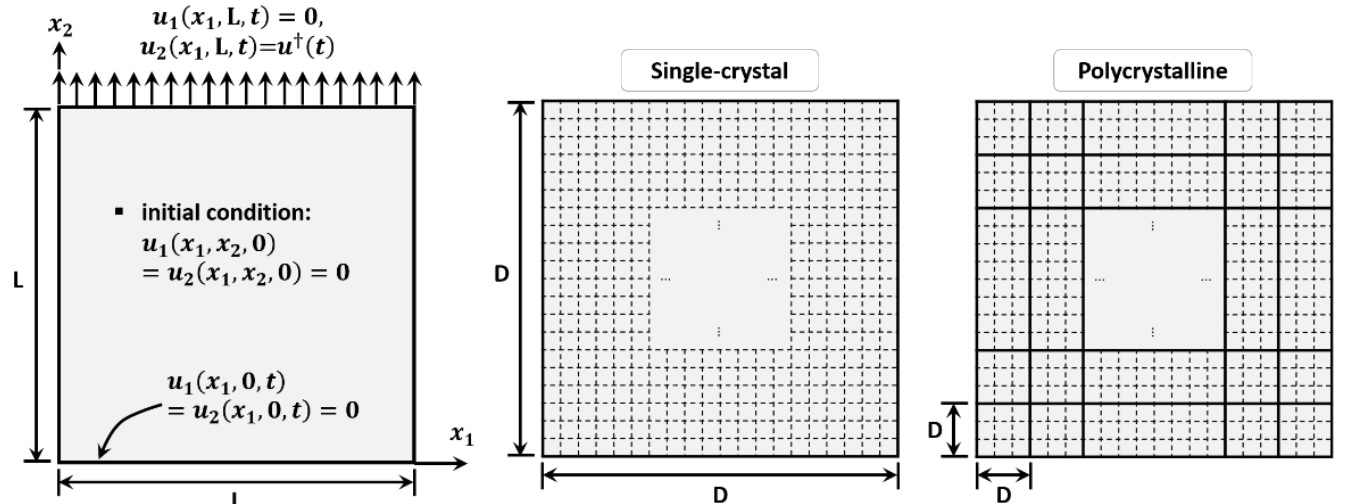

Figure 1. Schematic illustration of uniaxial strain problem with single-crystal and polycrystalline. D is the (average) grain size. Dotted lines and bold lines in single-crystal and polycrystalline represent finite element mesh and grain boundary respectively.

Table 1. Material properties used for the numerical simulations.

\begin{tabular}{clcc}
\hline & \multicolumn{1}{c}{ Parameters } & Units & Values \\
\hline$\mu$ & Shear modulus & $P a$ & $70.0 \mathrm{E} 9$ \\
$\kappa$ & Bulk modulus & $P a$ & $140.0 \mathrm{E} 9$ \\
$\rho$ & Density & $g \cdot \mathrm{cm}^{-3}$ & 2.702 \\
$h_{e n}$ & Energetic flow resistance parameter & $P a$ & $1.0 \mathrm{E} 0$ \\
$h_{d i s}$ & Dissipative flow resistance parameter & $P a$ & $5.0 \mathrm{E} 9$ \\
$\sigma_{y}^{0}$ & Initial yield strength for hardening/softening function & $P a$ & $100.0 \mathrm{E} 6$ \\
$\sigma_{y}^{s}$ & Initial strain hardening parameter for hardening/softening function & $P a$ & $100.0 \mathrm{E} 6$ \\
$\mathcal{H}$ & Initial strain softening parameter for hardening/softening function & $P a$ & $1.0 \mathrm{E} 9$ \\
$\varphi_{1}$ & Material parameter for hardening/softening function & - & 20.0 \\
$\varphi_{2}$ & Material parameter for damage variable & - & 0.0 \\
$\alpha^{t h}$ & Thermal expansion coefficient & $\mu m / m \cdot K$ & 16.4 \\
$\mathcal{T}_{r}$ & Reference temperature & $K$ & 298 \\
$n$ & Temperature sensitivity parameter & - & 0.7 \\
$\mathcal{T}_{y}$ & Thermal material parameter & $\mathrm{J} / \mathrm{g} \cdot{ }^{\circ} \mathrm{K}$ & 1,358 \\
$c_{\varepsilon}$ & Specific heat capacity at constant stress & $K^{-1}$ & 0.385 \\
$\theta_{0}$ & Flow stress softening parameter & $K^{-1}$ & 0.002 \\
$\theta_{\mathcal{H}}$ & Hardening/softening parameter & & 0.002 \\
\hline
\end{tabular}

\subsection{Hall-Petch strengthening}

Numerical simulations under the uniaxial tensile loading condition are carried out with six different grain sizes from 0.03125 to $1.0 \mathrm{~mm}$ to investigate the Hall-Petch strengthening and to validate the proposed model by comparing with the experimental data of [22]. The Hall-Petch constants such as $\sigma_{0}$ and $k$ are also studied in this section. The numerically obtained true stress-true strain curves are shown in Figure 2 with varying grain sizes. As can be seen in this figure, the Hall-Petch strengthening (material hardens with decreasing grain size) is well observed qualitatively.

The yield stress at a (true) strain of 0.002 and the flow stresses at four selected strains $(0.01,0.05,0.1,0.2)$ against the reciprocal square root of the grain size are plotted in Figure 3. Straight lines are fitted to the points by the least squares method. A significant grain size effect is predicted at the onset of yielding, which is in line with experimental finding. Both experimental data [22] and model predictions show a good linear correlation for all the grain sizes. Moreover, model predictions from the simulations are in an excellent agreement with experimental data. Table 2 shows the Hall-Petch constants, $\sigma_{0}$ and $k$, for both experiments and simulations at the different levels of strain obtained from the linear trendlines in Figure 3. Again, simulations show a good agreement with experiments in both parameters. 


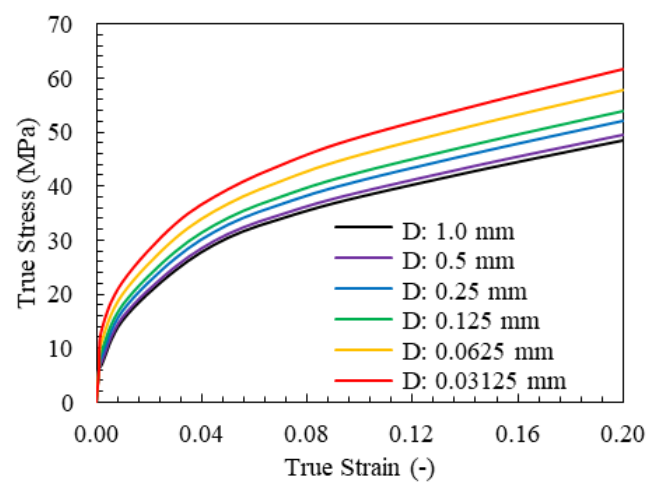

Figure 2. True stress-true strain responses with varying grain sizes from simulations.

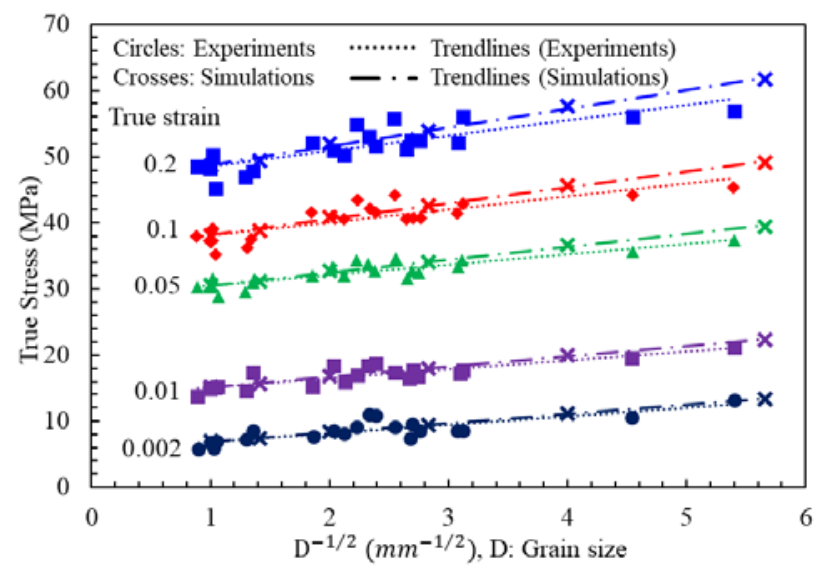

Figure 3. Hall-Petch plot for pure aluminium from experiments [22] and simulations.

Table 2. Hall-Petch constants for pure aluminium from experiments [22] and simulations.

\begin{tabular}{ccccc}
\hline \multirow{2}{*}{ True strain } & \multicolumn{2}{c}{ Experiments [22] } & \multicolumn{2}{c}{ Simulations } \\
\cline { 2 - 5 } & $\sigma_{0}(\mathrm{MPa})$ & $k\left(\mathrm{MPa} \cdot \mathrm{mm}^{1 / 2}\right)$ & $\sigma_{0}(\mathrm{MPa})$ & $k\left(\mathrm{MPa} \cdot \mathrm{mm}^{1 / 2}\right)$ \\
\hline 0.002 & 5.62 & 1.27 & 5.62 & 1.27 \\
0.01 & 13.83 & 1.39 & 13.83 & 1.34 \\
0.05 & 28.92 & 1.58 & 28.92 & 1.58 \\
0.1 & 36.15 & 1.95 & 36.15 & 1.95 \\
0.2 & 46.32 & 2.29 & 46.32 & 2.29 \\
\hline
\end{tabular}

Figure 3 and Table 2 imply that the Hall-Petch constants, $\sigma_{0}$ and $k$, increase with increasing strain. It is worth mentioning that the strengthening effect of the grain boundary areas described by $k$ increases with increasing strain.

These two parameters are plotted in Figure 4 as a function of strain. Besides the presented experimental data at the five strain levels in Figure 3 and Table 2, the other experimental data sets presented in [22] are also plotted in this figure. The good correlations between experiments and simulations are obtained.

This is in line with the findings of [23]. In [23], an empirical relationship of Hall [1] and Petch [2], Eq. (1), is extended by expressing the Hall-Petch constants, $\sigma_{0}$ and $k$, to depend on the strain level, such that $\sigma_{y}=\sigma_{0}(\varepsilon)+$ $k(\varepsilon) D^{-n}$ where the exponent $n$ typically ranges from 0.3 to 1.0 (the most reported value is 0.5 ).

Due to the formation of dislocation pile-ups at grain boundaries, flow stress can be enhanced and yielding occurs when flow stress is large enough to cause the slip to propagate from one grain to the adjacent grain. In order to underpin this behavior, physically based strain gradient plasticity models are in demand. The current model is phenomenological and physically based models have not been attained despite its importance. Furthermore, the pioneering measurements made by [24] were reported without assessment in terms of the Hall-Petch constants, $\sigma_{*}$ and $k$, but nevertheless show on examination decreasing values of both parameters with increase in temperature, more so in $\sigma_{*}$. A weak strain rate dependence of the flow stress was also measured by [24]. Effects of temperature and strain rate on the Hall-Petch constants will be investigated in the future. In addition, strain gradient crystal plasticity is another interest of the authors so that its pros and cons compared to the strain gradient continuum plasticity covered in this work will be explored in the future work. 


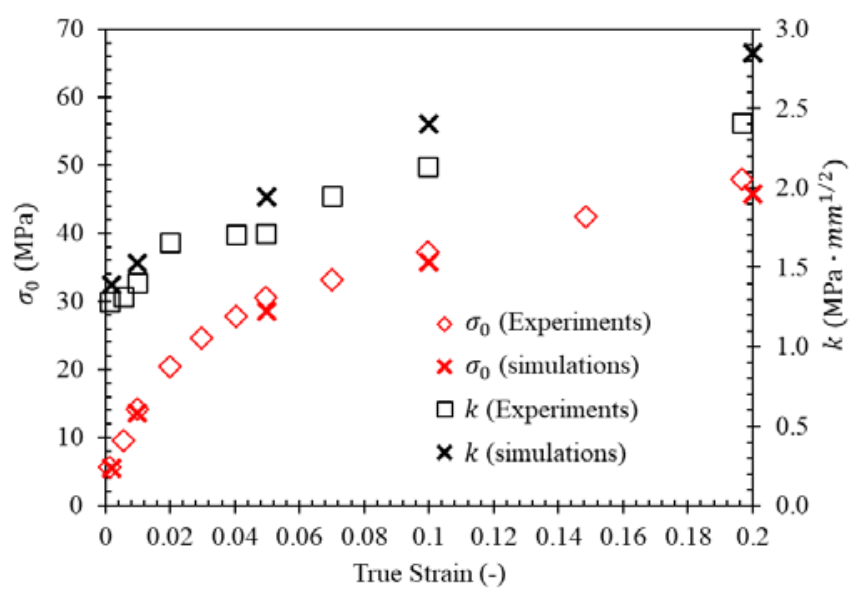

Figure 4. Hall-Petch constants as a function of strain for pure aluminum from experiments [22] and simulations.

\section{Conclusions}

The Hall-Petch relationship and effects of its strengthening on the flow stress in metals are investigated through the strain gradient plasticity model. The thermodynamically consistent gradient-enhanced plasticity flow rule is proposed correspondingly. The implementation of developed model is performed based on the finite element method. By comparing the obtained numerical results to the existing test data, the Hall-Petch relationship is studied. The two null boundary conditions, the microscopically free and hard boundary conditions, are well captured through the proposed model at the grain boundary. The six grain sizes are employed in this study and the HallPetch relationship is well described. The good correlations between the experimental measurements and the obtained simulation results are shown at the different strain levels. Finally, the Hall-Petch constants are shown to be changed with the strain level.

\section{References}

[1] Hall EO. The deformation and ageing of mild steel .3. Discussion of results. Proceedings of the Physical Society of London Section B. 1951;64(381):747-753.

[2] Petch NJ. The cleavage strength of polycrystals. Journal of the Iron and Steel Institute. 1953;174:25-28.

[3] Weertman JR. Hall-Petch strengthening in nanocrystalline metals. Materials Science and Engineering: A. 1993;166(1-2):161-167.

[4] Hansen N. Hall-Petch relation and boundary strengthening. Scripta Materialia. 2004;51(8):801-806.

[5] Armstrong RW. 60 years of Hall-Petch: past to present nano-scale connections. Materials Transactions. 2014;55(1):2-12.

[6] Pande CS, Cooper KP. Nanomechanics of Hall-Petch relationship in nanocrystalline materials. Progress in Materials Science. 2009;54(6):689-706.

[7] Evers LP, Parks DM, Brekelmans WA, Geers MG. Crystal plasticity model with enhanced hardening by geometrically necessary dislocation accumulation. Journal of the Mechanics and Physics of Solids. 2002;50(11):2403-2424.

[8] Voyiadjis GZ, Song Y. Effect of passivation on higher order gradient plasticity models for non-proportional loading: energetic and dissipative gradient components. Philosophical Magazine. 2017;97(5):318-345.

[9] Voyiadjis GZ, Song Y. Higher order thermo-mechanical gradient plasticity model: non-proportional loading with energetic and dissipative components. Springer International Publishing, Cham, Switzerland;2017.

[10] Voyiadjis GZ, Song Y, Park T. Higher-order thermomechanical gradient plasticity model with energetic and dissipative components. Journal of Engineering Materials and Technology. 2017;139(2).

[11] Song Y. A coupled thermo-mechanical theory of strain gradient plasticity for small and finite deformations. Louisiana State University, 2018.

[12] Song Y, Voyiadjis GZ. A two-dimensional finite element model of the grain boundary based on thermomechanical strain gradient plasticity. Journal of Theoretical and Applied Mechanics. 2018;56:377-391.

[13] Song Y, Voyiadjis GZ. Small scale volume formulation based on coupled thermo-mechanical gradient enhanced plasticity theory. International Journal of Solids and Structures. 2018;134:195-215. 
[14] Voyiadjis GZ, Song Y. Finite element analysis of thermodynamically consistent strain gradient plasticity theory and applications. Handbook of Nonlocal Continuum Mechanics for Materials and Structures. Springer International Publishing, Cham, Switzerland;2018. p. 1-58.

[15] Song Y, Yeon J, Na B. Numerical simulations of the hall-petch relationship in aluminium using gradientenhanced plasticity model. Advances in Civil Engineering. 2019;2019.

[16] Voyiadjis GZ, Song Y. Strain gradient continuum plasticity theories: theoretical, numerical and experimental investigations. International Journal of Plasticity. 2019;121:21-75.

[17] Gudmundson P. A unified treatment of strain gradient plasticity. Journal of the Mechanics and Physics of Solids. 2004;52(6):1379-1406.

[18] Gurtin ME, Anand L. Thermodynamics applied to gradient theories involving the accumulated plastic strain: the theories of Aifantis and Fleck and Hutchinson and their generalization. Journal of the Mechanics and Physics of Solids. 2009;57(3):405-421.

[19] Gurtin ME, Anand L. A theory of strain-gradient plasticity for isotropic, plastically irrotational materials. Part II: Finite deformations. International Journal of Plasticity. 2005;21(12):2297-2318.

[20] Voyiadjis GZ, Song Y. Higher order thermo-mechanical gradient plasticity model: non-proportional loading with energetic and dissipative components. In: G.Z. Voyiadjis (Ed.), Handbook of Nonlocal Continuum Mechanics for Materials and Structures. Springer International Publishing, Cham; 2017.p. 1-48.

[21] Voce E. Analysis of stress strain curves. The Journal of the Royal Aeronautical Society. 1955;59(534):442442.

[22] Hansen N. The effect of grain size and strain on the tensile flow stress of aluminium at room temperature. Acta Metallurgica. 1977;25(8):863-869.

[23] Armstrong RW, Codd I, Douthwaite RM, Petch NJ. The plastic deformation of polycrystalline aggregates. The Philosophical Magazine: A Journal of Theoretical Experimental and Applied Physics. 1962;7(73):45-58.

[24] Carreker RP, Hibbard WR. Tensile deformation of aluminum as a function of temperature, strain rate, and grain size. Transactions of the American Institute of Mining and Metallurgical Engineers. 1957;209:11571163.

(C) 2020 by the author(s). This work is licensed under a Creative Commons Attribution 4.0 International License (http://creativecommons.org/licenses/by/4.0/). Authors retain copyright of their work, with first publication rights granted to Tech Reviews Ltd. 